\title{
ABTS: A Transformation-Based Consistency Control Algorithm for Wide-Area Collaborative Applications
}

\author{
Bin Shao $^{\dagger}, \quad \mathrm{Du} \mathrm{Li}^{\ddagger}, \quad \mathrm{Ning} \mathrm{Gu}{ }^{\dagger}$ \\ $\uparrow$ School of Computer Science, Fudan University, Shanghai, China \\ $\ddagger$ Nokia Research Center, Palo Alto, California, USA \\ Email: binshao@fudan.edu.cn; lidu008@gmail.com; ninggu@ fudan.edu.cn
}

\begin{abstract}
Operational transformation (OT) is an established optimistic consistency control method in collaborative applications. Most existing OT algorithms are developed under a well-accepted framework with a condition that cannot be formally proved. In addition, they generally support two character-based primitive operations, insert and delete, in a linear data structure. This paper proposes a novel OT algorithm that addresses the above two challenges as follows: First, it is based on a recent theoretical framework with formal conditions such that its correctness can be proved. Secondly, it supports two string-based primitive operations and handles overlapping and splitting of operations. As a result, this algorithm can be applied in a wide range of practical collaborative applications.
\end{abstract}

\section{Introduction}

Operational transformation (OT) $[1,10]$ is an optimistic consistency control method that lies in the heart of many collaborative applications such as group editors [11] and Google Wave. ${ }^{1}$ The method replicates the shared data at cooperating sites. Local operations are always executed as soon as they are generated by the user. Remote operations are transformed before execution to repair inconsistencies. As a result, local responsiveness is not sensitive to communication latencies, which makes the method particularly appealing to collaborative applications running over widearea networks with high and nondeterministic latencies.

A plethora of OT algorithms have been proposed over the past two decades, e.g., $[4,9,10,11,12]$. There are two open challenges: First, most of them are developed under the framework of Sun et al [11], which includes an informal condition called "intention preservation". As a consequence, their correctness cannot be formally proved

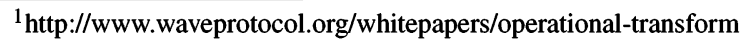

and counterexamples are often reported, as confirmed in $[4,7,5,8]$. Secondly, except for [11], all other OT algorithms only consider two character-based primitive operations. Although this simplification is theoretically acceptable, there is a practicality gap when applying those algorithms to real collaborative applications in which stringbased operations are common. The handling of string operations is very intricate, as confirmed in [11].

To address the above two challenges, this paper proposes a novel OT algorithm called Admissibility-Based Transformation with Strings (ABTS): First, it is based on the ABT framework $[6,5]$ which formalizes two correctness condition, causality and admissibility preservation. Conceptually, admissibility requires that the execution of every operation not violate the relative position of effects produced by operations that have been executed so far. As a result, the original ABT algorithm and the derived ABTS algorithm can be formally proved. Secondly, ABTS supports two string-based primitive operations and their overlapping and splitting when concurrent operations are transformed. As a result, the algorithm can be directly applied in a range of collaborative applications that require string operations. Moreover, the design of ABTS will provide a new starting point when extending OT algorithms to support composite and block operations that semantically must be applied together, such as cut-paste and find-replace.

Section 2 gives the background of this research. Section 3 introduces notations. Section 4 presents the ABTS algorithm. Sections 5 and 6 analyze its correctness and complexities, respectively. Finally, Section 7 concludes.

\section{Background and Related Work}

To illustrate the basic ideas of OT, consider a scenario in which two users, A and B, collaboratively edit a shared document which includes a list of guests to invite for a party. The document is replicated at the two sites when the users discuss about it online. Suppose that the list is ini- 
tially "Tom" and the first position of a string is zero. User A extends the list to "Karen,Tom" by operation $o_{A}=$ insert ( 0 , "Karen,"). At the same time, user B extends the list to "Tom,Sarah" by operation $O_{B}=\operatorname{insert}(3$, ,'Sarah”). The two sites diverge before their results are merged.

When A receives $o_{B}$, if the operation were executed asis, the wrong result"Kar,Sarahen,Tom" would yield in the list of A. The intuition of OT [1] is to transform remote operations to incorporate the effects of concurrent local operations that have been executed earlier. In this scenario, for example, A transforms $o_{B}$ into a form $o_{B}^{\prime}$ such that $o_{B}^{\prime}$ can be correctly executed in current state "Karen,Tom" of site A. Considering the fact that $A$ has inserted a string of six characters on the left side of the intended position of $o_{B}$, we must shift the position of $o_{B}$ by six to the right, yielding $o_{B}^{\prime}$ $=\operatorname{insert}$ (9, "Sarah"). Execution of $o_{B}^{\prime}$ in state "Karen,Tom" results in the right list of "Karen,Tom,Sarah". On the other hand, when user B receives $o_{A}$, the operation can be executed as-is in current state of $B$ because the target position of $o_{A}$ is not affected by the execution of $o_{B}$. This results in list "Karen,Tom,Sarah". Now the two sites converge.

The philosophy of OT is to avoid operation overwriting so as not to lose user interaction results. To reduce chaos in the result as caused by concurrency, Sun et al [11] propose three conditions to constrain the output, namely, convergence, causality and intention preservation. Unfortunately, although intuitive and widely accepted, intention preservation is not a well-formalized condition. As a consequence, OT algorithms developed under their framework (e.g., $[10,11,9])$ are not completely proved and counterexamples are often reported $[4,7,5,8]$.

Our work has established formal, provable correctness conditions $[4,7,6]$. In particular, the Admissibility-Based Transformation (ABT) framework $[6,5]$ proposes an alternative constraint called admissibility, which conceptually requires that the execution of any operation not violate the character order established by previous executions in the system. However, they mainly serve theoretical purposes and only consider two characterwise primitives. The presented stringwise ABTS algorithm is a significant extension to its characterwise version ABT $[6,5]$. Specifically, when transforming two stringwise operations, the algorithm is greatly complicated by the handling of position relations between the operation regions because operations may be split cascadingly during transformation.

In the literature, only the GOT algorithm [11] supports stringwise operations. In their follow-up work (e.g., $[10,12])$, new algorithms are proposed to replace GOT. We believe that they have implemented stringwise operations. However, in their publications, they have not addressed how to prove the correctness of their algorithms and how to support stringwise operations in their new algorithms. Note that this is not to say that their algorithms are incorrect.
Nevertheless, GOT converges by maintaining a predefined total order of operations and using a do/undo/redo based control procedure integrated with OT. In order to utilize the do/undo/redo mechanism, GOT requires that all operations be reversible. To ensure reversibility, a "lost and found" mechanism is employed to save and restore the object relations between two operations in transformation. Hence its space complexity is $O\left(|H|^{2}\right)$, where $H$ is the history buffer. By comparison, ABTS requires neither a total order of execution nor reversibility of operations; correctness is ensured without saving the object relation; its space complexity is $O(|H|)$. We prefer not to compare the time complexity of GOT before all its details are presented, e.g., their solutions to the counterexample identified in [4].

\section{System Model and Notations}

A system consists of a number of collaborating sites. The same version of the shared data is replicated at all sites when a session starts. For local responsiveness, each site submits operations only to its local replica which are executed immediately. In the background, local operations are propagated to remote sites.

The shared data is abstracted as a linear string of atomic characters. Objects are referred to by their positions in the string, starting from zero. For simplicity, we consider two primitive operations, namely, $\operatorname{insert}(p, s)$ and delete $(p, s)$, which insert and delete a string $s$ at position $p$ in the shared data, respectively. Any operation $o$ has the following attributes: o.id is the unique id of the site that originally submits $o$; o.type is the operation type which is either insert or delete; o.pos is the position in the shared data at which $o$ is applied; o.str is the target string which the operation inserts or deletes. We use established notations [2] happens-before $(\rightarrow)$ and concurrent $(\|)$ to denote the temporal relations between operations.

Note that, for any operation o, o.pos is always defined relative to some specific state of the shared data. Following notations in [10], the definition state of $o$, denoted as $\operatorname{dst}(o)$, is the state in which o.pos is defined. Given any two operations $o_{1}$ and $o_{2}$, we say that they are contextually equivalent, denoted as $o_{1} \sqcup o_{2}$, if $\operatorname{dst}\left(o_{1}\right)=\operatorname{dst}\left(o_{2}\right)$; they are contextually serialized, denoted as $o_{1} \mapsto o_{2}$, if $o_{2}$ 's position is defined in the resulting state of applying $o_{1}$ (but no other operation). For example, in the scenario given in Section 2, we have $o_{A} \| o_{B}, o_{A} \sqcup o_{B}$, and $o_{A} \mapsto o_{B}^{\prime}$.

A list is an ordered collection of objects, denoted as $\left[e_{1}, \ldots, e_{n}\right]$. An empty list is denoted as []. For any list $L$, notation $|L|$ denotes the number of objects in $L$ and $|[]|$ $=0$. Borrowing notations from Prolog, if $|L|>0$, L.head refers to its first element $L[0]$, and L.tail refers to remaining list $L[1 . .|L|-1]$. For example, if $\mathrm{L}=[\mathrm{a}, \mathrm{b}, \mathrm{c}]$, then $\mathrm{L}$.head $=\mathrm{a}$ and L.tail $=[\mathrm{b}, \mathrm{c}]$. Operator $\cdot$ concatenates two lists, or a 


\begin{tabular}{|l|l|}
\hline Notation & Brief Description \\
\hline \hline o.id & the id of site that originally generates $o$ \\
o.type & $\begin{array}{l}\text { the operation type of } o \text {, either } i n s \text { or } \text { del } \\
\text { the position of } o \text { relative to the data model }\end{array}$ \\
$o . s t r$ & the string inserted or deleted by $o$ \\
\hline$o_{1} \rightarrow o_{2}$ & $o_{1}$ happens before $o_{2}$ \\
$o_{1} \| o_{2}$ & $o_{1}$ and $o_{2}$ are concurrent \\
$o_{1} \sqcup o_{2}$ & $o_{1}$ and $o_{2}$ are contextual equivalent \\
$o_{1} \mapsto o_{2}$ & $o_{1}$ and $o_{2}$ are contextually serialized \\
\hline$\left[o_{1}, o_{2}\right]$ & an ordered list of two operations \\
$<o_{1}, o_{2}>$ & a 2-operation sequence in which $o_{1} \mapsto o_{2}$ \\
$|L|$ & the number of objects in list/sequence $L$ \\
$L_{1} \cdot L_{2}$ & a concatenated list/seq of two lists/seqs \\
\hline
\end{tabular}

Table 1. A summary of the main notations.

list and an object. For example, for $L=[a, b, c]$, we have $L$ $=[a] \cdot[b, c]=[a, b] \cdot c$.

A sequence is a special list in which all elements are operations that are contextually serialized. A sequence $s q$ of $n$ operations is denoted as $s q=<o_{1}, o_{2}, \ldots, o_{n}>$, where $o_{1} \mapsto o_{2} \mapsto \ldots \mapsto o_{n}$. An empty sequence is denoted as $<>$. The above list notations also apply to sequences, e.g., $|s q|=n$ and $s q=<o_{1}>\cdot<o_{2}, \ldots o_{n}>$.

\section{The ABTS Algorithm}

We first overview the ABTS algorithm and then explain the involved procedures in the following subsections.

\subsection{Overview}

A history buffer $H$ is maintained at each site which logs operations that have been applied to the data replica at that site. For correctness reasons $[6,5], H$ is maintained as a concatenation of of two subsequences, $H_{i}$ and $H_{d}$, which record the executed insert and delete operations in their order of execution, respectively. That is, $H=H_{i} \cdot H_{d}$. In addition, each site maintains $R Q$, a list of operations received from remote sites in their order of arrival. Each site $j$ runs the following three concurrent threads:

Thread $\mathcal{L}$ each time receives a local operation $o$, applies it to the data replica, calls algorithm update $H L$ to update $H$ and compute $o^{\prime}$, a transformed version of $o$, and propagates the resulting $o^{\prime}$ to remote sites.

Thread $\mathcal{N}$ receives remote operations from the network and appends them to $R Q$ in their order of arrival.

Thread $\mathcal{R}$ scans $R Q$ for a remote operation $o$ at a time that is causally-ready, i.e., all operations that happen before $o$ have been executed at site $j$. Then algorithm update $H R$ is called to update $H$ and transform $o$ into a version $o^{\prime}$ that can be correctly executed in current state of site $j$. After that, $o^{\prime}$ is executed on the data replica at site $j$.

\subsection{Algorithm updateHL}

By the way $H$ is maintained, a new local insertion $o_{i}$ must be appended to $H_{i}$ and a new deletion $o_{d}$ to $H_{d}$. Note that all operations that have been executed on the local data replica are included in $H$; the new local operation $o$ ( $o_{i}$ or $o_{d}$ ) is defined in the current state of the shared data. That is, all operations in $H$ happen before $o$ (or $H \rightarrow o$ ); $H$ and $o$ are contextually serialized (or $H \mapsto o$ ). Hence, we can directly append $o_{d}$ to $H_{d}$ because $H_{d} \mapsto o_{d}$ also holds. However, we cannot directly append $o_{i}$ to $H_{i}$ because $H_{i} \mapsto$ $o_{i}$ does not hold due to the existence of $H_{d}$.

We solve this problem by computing $o_{i}^{\prime}$, some version of $o_{i}$, such that $H_{i} \mapsto o_{i}^{\prime}$. This is achieved by swapping $H_{d}$ and $o_{i}$. Before the swapping, $H_{d} \mapsto o_{i}$. As a result of the swapping, they become $H_{d}^{\prime}$ and $o_{i}^{\prime}$, respectively, such that $o_{i}^{\prime} \mapsto H_{d}^{\prime}$. Then $o_{i}^{\prime}$ can be appended to $H_{i}$.

To explain swapping, consider a scenario with initial state "xy". First execute $o_{1}=\operatorname{delete}(1$, ' $y$ ') to reach state " $\mathrm{x}$ ". Then execute $o_{2}=\operatorname{insert}(0$, ' $\mathrm{z}$ ') to yield state " $\mathrm{zx}$ ". The relation is $o_{1} \mapsto o_{2}$. If we swap $o_{1}$ and $o_{2}$, yielding $o_{1}^{\prime}$ and $o_{2}^{\prime}$, respectively, such that $o_{2}^{\prime} \mapsto o_{1}^{\prime}$, it must be that $o_{2}^{\prime}=\operatorname{insert}\left(0,{ }^{\prime} \mathrm{z}\right)$ and $o_{1}^{\prime}=\operatorname{delete}\left(2, \mathrm{y}^{\prime}\right)$. As a result, $o_{2}^{\prime}$ is executed before $o_{1}^{\prime}$ yet they produce the same effects.

Function updateHL $(o)$ not only appends $o$ to the right subsequence but also its swapping process excludes the effects of $H_{d}$ from $o$ as if no deletions had been executed before $o$ at current site. In either case of $o\left(o_{i}\right.$ or $\left.o_{d}\right)$, after swapping $o$ and $H_{d}$, we obtain $o^{\prime} \mapsto H_{d}^{\prime}$. As a result, none of the deletion effects of $H_{d}$ are included in the definition state of $o^{\prime}$ when it is propagated to remote sites.

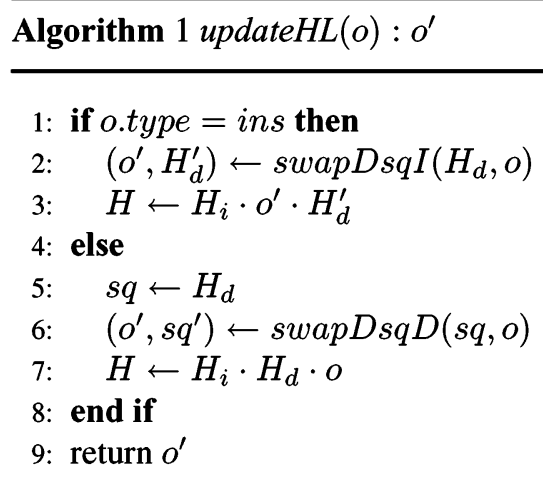

Based on the above explanation, Algorithm 1 specifies function updateHL, which works as follows: If the new local operation $o$ is an insertion, we swap it with $H_{d}$ and append the resulting $o^{\prime}$ to $H_{i}$. Then we update the history to $H_{i} \cdot o^{\prime} \cdot H_{d}^{\prime}$. The resulting $o^{\prime}$ is returned (line 9) and will be propagated to remote sites. On the other hand, if $o$ is a deletion, we directly append it to subsequence $H_{d}$ (line 7) and update the history to $H_{i} \cdot H_{d} \cdot o$. Meanwhile we exclude 
the effects of $H_{d}$ from $o$ by swapping $o$ with a copy of $H_{d}$ (lines 5-6). Then the resulting $o^{\prime}$ is returned and propagated to remote sites. The two swapping procedures, swapDsqI and swapDsqD, will be presented later in Section 4.6.

\subsection{Algorithm updateHR}

Function updateHR $(o)$ has two goals, where $o$ is a causally-ready remote operation: First, it appends $o$ to $H_{i}$ or $H_{d}$ depending on o.type. Secondly, it outputs $o^{\prime}$, a version of $o$, such that $o^{\prime}$ can be executed in current state.

When updateHR $(o)$ is called in thread $\mathcal{R}$, operation $o$ must be causally ready. That is, all operations that happen before $o$ are already executed and included in the history $H=H_{i} \cdot H_{d}$. However, note that some operations that are concurrent with $o$ may also have been executed at current site. That is, subsequences $H_{i}$ and $H_{d}$ include both operations that happen before $o$ and those concurrent with $o$.

The intuition of OT [1] is to transform an operation $o$ with those with effects not included in $o$ to incorporate their effects. This process is called inclusion transformation or IT [10]. For example, in the scenario in Section 2, by step $o_{B}^{\prime}=\operatorname{ITT}\left(o_{B}, o_{A}\right)$, operation $o_{B}$ is inclusively transformed with $o_{A}$ to incorporate the effect of $o_{A}$. The relationship between the two operations is $o_{B} \sqcup o_{A}$ before the IT step and $o_{A} \mapsto o_{B}^{\prime}$ after. Then the resulting $o_{B}^{\prime}$ can be correctly executed in current state of site $A$.

In fact, we do not need to transform $o$ with all operations in $H$ because the effects of some of operations in $H_{i}$ that happen before $o$ are already included in $o$. To distinguish, we must somehow transpose $H_{i}$ into two contextually serialized subsequences $s q_{h}$ and $s q_{c}$, such that $H_{i}=s q_{h} \cdot s q_{c}$, where $s q_{h}$ contains all operations in $H_{i}$ that happen before $o$ and $s q_{c}$ contains all operations in $H_{i}$ that are concurrent with $o$. Then history $H$ is equivalent to $s q_{h} \cdot s q_{c} \cdot H_{d}$.

According to Section 4.2, before $o$ is propagated, it has excluded the effects of all deletions that happen before it; however, it includes the effects of all insertions that happen before it, which are exactly all the operations in subsequence $s q_{h}$. Hence $o$ does not include any effects in subsequence $s q_{c} \cdot H_{d}$. Then $s q_{h} \mapsto o$ and $o \sqcup\left(s q_{c} \cdot H_{d}\right)$. If we get $o^{\prime}$ by inclusively transforming $o$ with $s q_{c} \cdot H_{d}$, then $o^{\prime}$ can be executed in current state. After the transformation, the relationship will be $\left(s q_{c} \cdot H_{d}\right) \mapsto o^{\prime}$ and $H \mapsto o^{\prime}$.

Now consider the goal of how to add $o$ to $H$. If $o$ is a deletion, then $o^{\prime}$ can be directly appended to $H_{d}$. However, if $o$ is an insertion, we only need to transform $o$ with $s q_{c}$ to get $o^{\prime \prime}$ and append $o^{\prime \prime}$ to $H_{i}$. Nevertheless, we cannot naively add $o^{\prime \prime}$ between $H_{i}$ and $H_{d}$ because, although $H_{i} \mapsto o^{\prime \prime}$, the relationship is $o^{\prime \prime} \sqcup H_{d}$ rather than $o^{\prime \prime} \mapsto H_{d}$. Therefore, we must first transform all operations in $H_{d}$ to incorporate the effect of $o^{\prime \prime}$, yielding $H_{d}^{\prime}$, and then update the history $H$ to $H_{i} \cdot o^{\prime \prime} \cdot H_{d}^{\prime}$.



As in Algorithm 2, we specify function updateHR based on the above discussions. In line 1 , it first transposes $H_{i}$ into two contextually serialized subsequences, $s q_{h}$ and $s q_{c}$, by calling algorithm transposeHC. In line 2 , it calls algorithm ITOSq to get $o^{\prime \prime}$ by transforming $o$ with $s q_{c}$. Then in line 3, it calls algorithm ITOSq again to get $o^{\prime}$ by transforming $o^{\prime \prime}$ with $H_{d}$. If $o$ is an insertion, it transforms $H_{d}$ to incorporate the effect of $o^{\prime \prime}$. After that, $o^{\prime \prime}$ is added between $H_{i}$ and the resulting $H_{d}^{\prime}$. If $o$ is a deletion, it simply appends $o^{\prime}$ to $H_{d}$. Finally, $o^{\prime}$ is returned and executed by thread $\mathcal{R}$ in the current state of the shared data.

Algorithm transposeHC is already well-understood [4, 6, $9,10]$ and here omitted. The two IT functions, ITOSq and ITDsqI, will be explained in Section 4.5.

\subsection{Atomic and Composite Operations}

To support stringwise transformation, we need to introduce a few more notations. Given any string $s$, notation $|s|$ is the number of characters in $s$. If $0 \leq i<j \leq|s|$, notation $s[i: j]$ returns a substring of $s$ starting from position $i$ to position $j-1$. If $j$ is not specified, $s[i$ : ] returns a substring from $i$ to the end. For example, let $s=$ "abc", then $|s|=3$ and $s[0: 2]=" a b "$ and $s[1:]=" b c "$.

A stringwise operation can be denoted as a list of suboperations that achieves the same effects. We use notation $o . s o l$ to denote the sub-operation list of operation $o$. Notation $o . s o l[i]$ is simplified as $o[i]$, and $|o . s o l|$ as $|o|$. For an atomic operation $o$, its sub-operation list only includes itself or $|o|=1$. A composite operation $o$ has more than one sub-operation or $|o|>1$. Operations in $o . s o l$ have the same $i d$ and type properties (as well as timestamps) but different positions. We extend operation relations such as $\rightarrow, \|, \sqcup$, and $\mapsto$ to composite operations without re-definition.

Here we are only interested in sub-operations of deletions. For example, given string "abc", delete( 0 , "abc") can be denoted as a list of two sub-operations, delete ( 0 , "a") and delete( 1 , "bc"). As another example, if for some reason we need to delete ' $a$ ' and ' $c$ ' in conceptually one operation, we 
may define a composite operation with two sub-operations delete $\left(0,{ }^{\prime} a\right.$ ') and delete $\left(2,{ }^{\prime} c\right)$. Note that positions of all operations in $o . s o l$ are defined relative to the same state, $\operatorname{dst}(o)$. That is, they are contextually equivalent with regard to $\operatorname{dst}(o)$. Hence, to achieve the same effects as $o$, they must be applied simultaneously to $\operatorname{dst}(o)$. It would be wrong to apply them one after another like a sequence.

As will be shown in Sections 4.5 and 4.6, when a deletion is transformed with another insert or delete operation, the result could be a composite deletion with two or more suboperations. Hence, the (delete) operations being propagated or received could be composite operations.

Algorithm 3 specifies the function for executing (atomic and composite) operations. In particular, to execute a composite operation $o$, we need to do a special transformation to o.sol before applying the sub-operations in $o . s o l$ in tandem. Algorithm 4 specifies this special transformation, called selfIT, which only adjusts positions of sub-operations that belong to the same composite operation $o$. Assume that an atomic operation's sub-operation list only includes itself. The algorithm first initializes sol with the given $o . s o l$; then for every sub-operation $\operatorname{sol}[i]$, subtract the total length of substrings deleted by preceding operations ranging from sol $[0]$ to $\operatorname{sol}[i-1]$. As a result, every $\operatorname{sol}[i]$ has accounted for the effects of preceding operations in the list. Then, operations in the resulting sol list (actually now a sequence) can be executed one by one in $\operatorname{dst}(o)$.

\begin{tabular}{l}
\hline Algorithm 3 execute $(o)$ \\
\hline 1: sol $\leftarrow \operatorname{selfIT}(o)$ \\
2: for $(i=0 ; i<\mid$ sol $\mid ; i++)$ do \\
3: apply $\operatorname{sol}[i]$ in shared data \\
4: end for
\end{tabular}

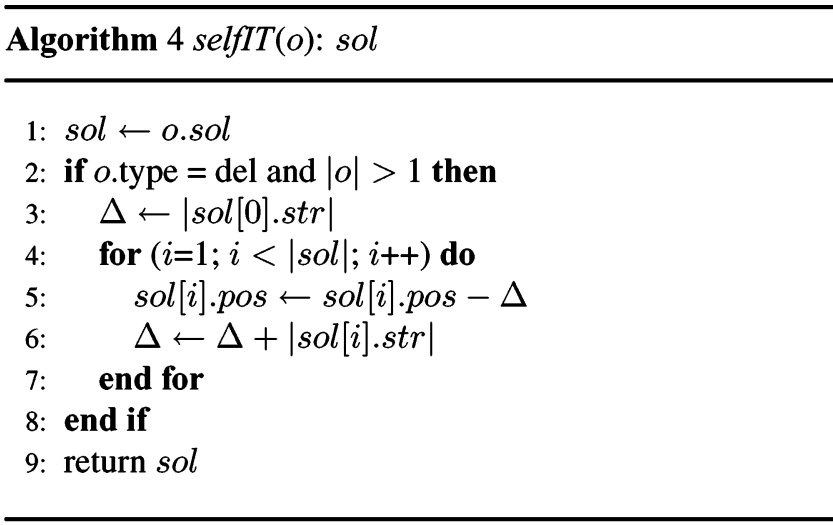

Based on selfIT, we could define the following two simple utility functions: getSubOpList(sq) collects a list of sub-operations of all operations in a given sequence $s q$, after applying selfIT on every $s q[i]$; and function
combineSubOpList(ol) returns a sequence of composite operations by combining all their sub-operations in a given list ol. These two functions are inverse of each other. For space reasons, we leave out their specifications in this paper.

\subsection{IT Algorithms}

In this subsection, we first discuss the most basic IT functions and then discuss advanced IT functions that involve at least one list (sequence) of primitive operations.

\subsubsection{Basic IT Functions}

In the most basic form, function $\operatorname{IT}\left(o_{1}, o_{2}\right)$ transforms a primitive operation $o_{1}$ with another primitive operation $o_{2}$ and outputs result $o_{1}^{\prime}$. As will be shown shortly, the output result is sometimes a composite operation. By the types of the two involved operations, insert (I) and delete (D), we define four functions, ITII, ITID, ITDI, and ITDD, as in Algorithms 5-8, respectively. According to [10], the precondition of $\operatorname{IT}\left(o_{1}, o_{2}\right)$ is $o_{1} \sqcup o_{2}$ and the postcondition is $o_{2} \mapsto o_{1}^{\prime}$. Intuitively, the positions of two operations must be defined in the same state so as to be compared in transformation. We will discuss the precondition further in Section 5.

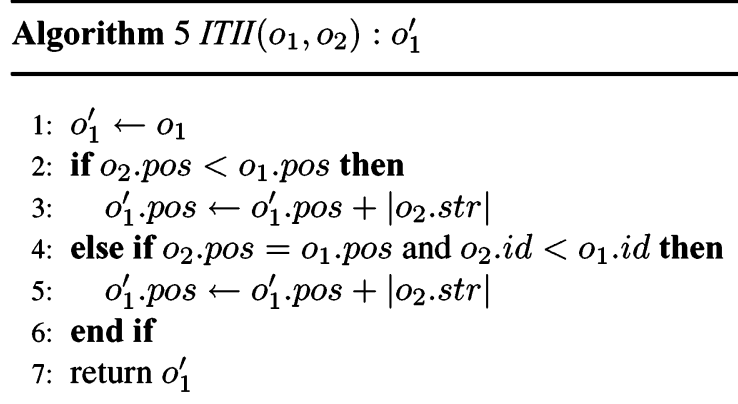

Algorithm $6 \operatorname{ITID}\left(o_{1}, o_{2}\right): o_{1}^{\prime}$



Algorithm 5 transforms insertion $o_{1}$ with another insertion $O_{2}$ to incorporate the effects of $o_{2}$. As shown in the condition of line 2, if $o_{2}$.pos is on the left of $o_{1}$.pos, meaning $o_{1} . s t r$ is to be inserted after $o_{2} . s t r$ is inserted, then $o_{1}$.pos should be shifted to the right by $\left|o_{2} . s t r\right|$ characters. 
If $o_{1}$.pos and $o_{2}$.pos tie, however, we use a priority scheme, e.g., by comparing their site ids, to break the tie: the two strings are ordered by their site ids in the result. That is, if $o_{1}$.id is greater, $o_{1}$.pos is shifted to the right. An intuitive scenario is that two users concurrently insert two strings at the same position in the same state. Using site ids to break the tie is a reasonable resort in concurrency control.

In Algorithm 6, an insertion $o_{1}$ is transformed with a deletion $o_{2}$. Let $s$ be their common definition state. Since $o_{2}$ deletes a substring that is already in $s$ and $o_{1}$ is to insert a new string into $s$, the deletion affects $o_{1}$.pos only when $o_{1}$.pos $>o_{2}$.pos, as shown in the condition of line 2. There are two cases: $o_{1}$.pos may fall out the right border of $o_{2} . s t r$ or within it. The condition in line 3 handles the former case, in which $o_{1}$.pos is shifted to the left by $\left|o_{2} . s t r\right|$ characters. Otherwise, $o_{1}$ is to insert within a substring that is deleted by $o_{2}$. Note that, in this case, either policy is reasonable: keep $o_{1}$.str or remove $o_{1}$.str. In this paper, we choose to keep $o_{1}$.str in the result and hence reset its new position to be the same as that of $o_{2}$, as shown in lines 5-6.

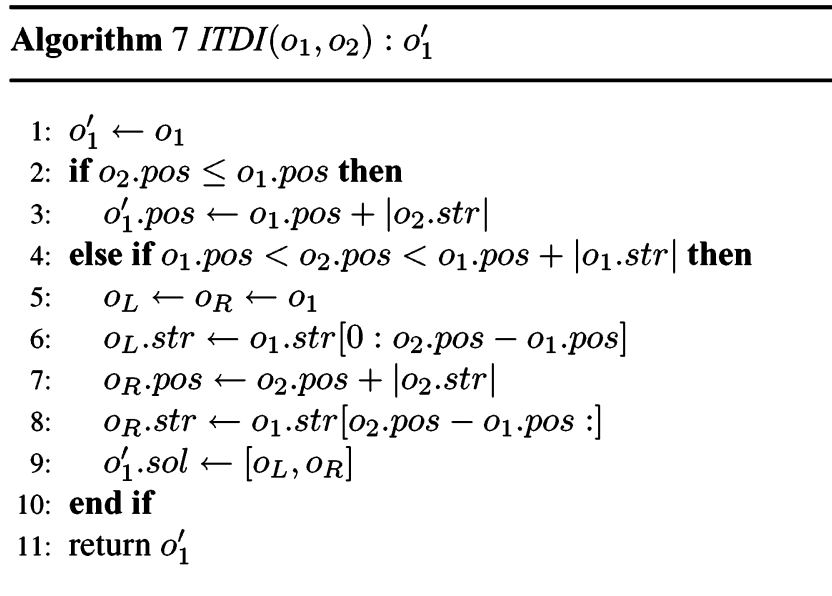

Algorithm 7 specifies how to transform a deletion $o_{1}$ with an insertion $o_{2}$. Let $s$ be their common definition state. Since $o_{1} . s t r$ is already in $s$ and $o_{2}$ inserts new content into $s$, we can use $o_{1} . s t r$ as the reference. As in the condition of line 2, if $o_{2}$ inserts on the left of $o_{1}$.pos, we need to shift $o_{1}$.pos by $\left|o_{2} . s t r\right|$ characters to the right. However, if $o_{2}$ inserts within the substring that $o_{1}$ intends to delete, as in the condition of line 4 , we need to split $o_{1} . s t r$ into two parts that are separated by $o_{2} . s t r$. As shown in lines 5-9, the transformation result is a composite operation with two sub-operations: $o_{L}$ deletes the substring up to the position pointed to by $o_{2}$.pos (exclusively), starting at position $o_{1}$.pos in $s$; and $o_{R}$ deletes the substring inclusively from after $o_{2} . p o s$, starting at the position in $s$ right after $o_{2} . s t r$, that is, $o_{2} . p o s+\left|o_{2} . s t r\right|$.

As shown in Algorithm 8, transforming two deletions is more complicated. Both $o_{1}$ and $o_{2}$ are to delete an existing

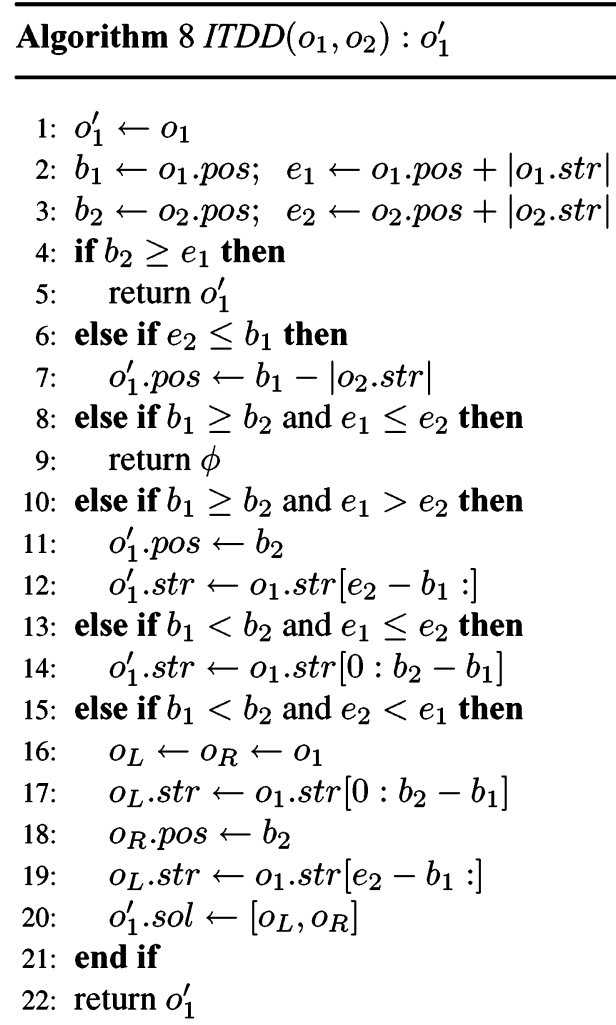

substring in their definition state $s$. We need to consider the following six cases regarding the relations between the two target regions, $R_{1}=s\left[b_{1}: e_{1}\right]$ and $R_{2}=s\left[b_{2}: e_{2}\right]$.

1. (line 4) $R_{2}$ is completely on the right of $R_{1}$. Deletion of $R_{2}$ does not affect $o_{1}$. Hence $o_{1}$ is returned as-is.

2. (line 6) $R_{1}$ is on the right of $R_{2}$. After $R_{2}$ is deleted, we shift $o_{1}$.pos by $\left|o_{2} . s t r\right|$ characters to the left.

3. (line 8) $R_{1}$ is included in $R_{2}$. Hence after $o_{2}$ is executed, $R_{1}$ is already deleted. There is no longer need to execute $o_{1}$. We return an empty operation $\phi$.

4. (line 10) $R_{2}$ partially overlaps with $R_{1}$ around the left border of $R_{1}$. After $o_{2}$ is executed, the left part of $R_{1}$ is already deleted. Hence, we need to reset $o_{1}$.pos so that it will start from $b_{2}$. And $o_{1}$.str only needs to include the right part that is not deleted by $o_{2}$, starting from $e_{2}-b_{1}$ in the original $o_{1} . s t r$.

5. (line 13) $R_{2}$ partially overlaps with $R_{1}$ around the righ border of $R_{1}$. This case similar to case (4). After $o_{2}$ is executed, $o_{1}$ only needs to delete the left part that is not deleted by $o_{2}$.

6. (line 15) $R_{2}$ is included in $R_{1}$. This case is similar to the case of lines 4-10 in Algorithm 7. The deletion of $R_{2}$ within $R_{1}$ divides $R_{1}$ into three parts, among which the middle overlapping part is already deleted by $o_{2}$. Hence $o_{1}$ must be split into two sub-operations that delete the two remaining substrings, respectively. 


\subsubsection{Sequence-Related IT Functions}

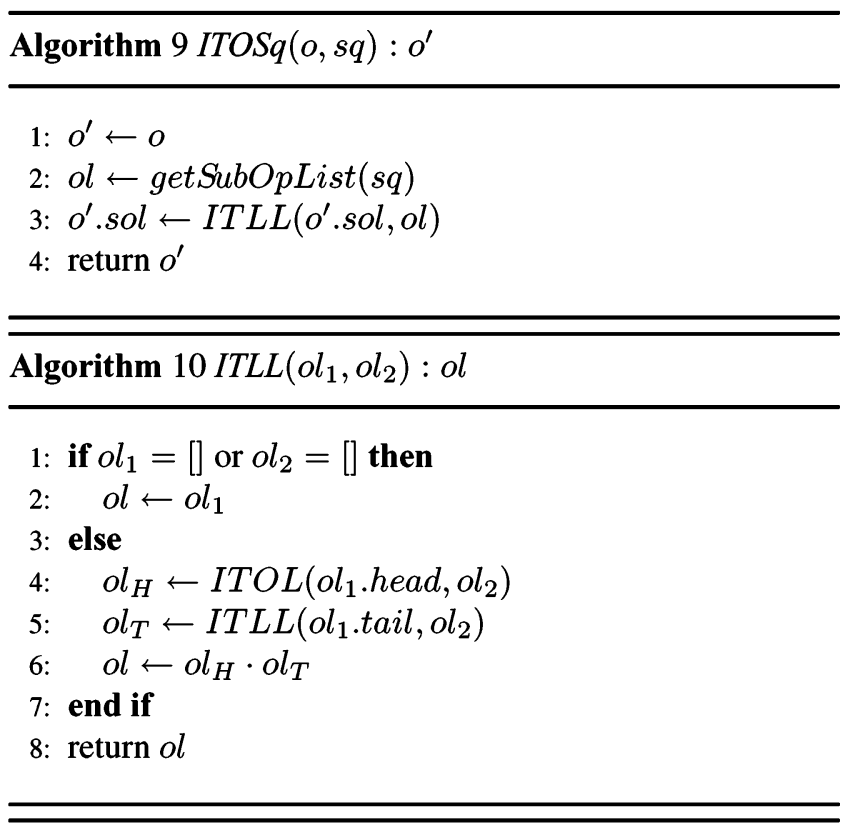

$$
\text { Algorithm } 11 \text { ITOL }\left(o, o_{2}\right): o l
$$

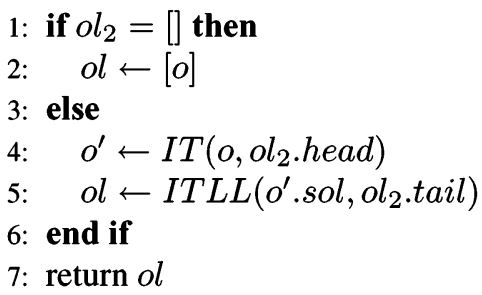

Now we specify the two functions, ITOSq and ITDsqI, that are used in function updateHR (Algorithm 2). As shown in Algorithm 9, function ITOSq $(o, s q)$ transforms an operation $o$ with a sequence $s q$. To do that, we need to transform $o$ one by one with every operation $s q[i]$. However, both $o$ and $s q[i]$ could be composite. To simplify processing, we first collect all sub-operations of $s q$ in list $o l$ by calling function getSubOpList(sq). Then, we use another algorithm to transform one list $o . s o l$ with another list $o l$.

The algorithm to transform two lists is implemented by a double recursion of two functions, ITLL and ITOL. As shown in Algorithm 10, ITLL $\left(o l_{1}, o l_{2}\right)$ transforms one list $o l_{1}$ with another list $o l_{2}$. In the simplest case, if $o l_{1}$ or $o l_{2}$ is empty, just return $o l_{1}$. Otherwise, we divide $o l_{1}$ into two parts, its first element $o l_{1}$.head and the rest of the list $o l_{1}$.tail. Then we call function ITOL to transform operation $o l_{1}$.head with list $o l_{2}$, yielding $o l_{H}$, and call function ITLL to transform list $\mathrm{ol}_{1}$.tail with list $\mathrm{ol}_{2}$, yielding $\mathrm{ol}_{T}$. Finally, we concatenate these two partial results, $o l_{H}$ and $o l_{T}$, and return $o l_{H} \cdot o l_{T}$ as the result of ITLL $\left(o l_{1}, o l_{2}\right)$.
In Algorithm 11, function ITOL $\left(o, o l_{2}\right)$ transforms an operation with a list $\mathrm{ol}_{2}$. Given a non-empty list $\mathrm{ol}_{2}$, we need to transform $o$ with operations in $o l_{2}$ one by one. Depending on types of the two involved operations, we first call some IT $\left(o, o l_{2} . h e a d\right)$ to transform $o$ with the first operation in $\mathrm{ol}_{2}$, yielding intermediate result $o^{\prime}$; then we call $\operatorname{ITLL}\left(o^{\prime}\right.$. sol, ol $o_{2}$.tail $)$ to transform $o^{\prime} . s o l$ with the rest of list $\mathrm{ol}_{2}$. Note that the intermediate list $o^{\prime}$. sol may be a singleton if the result is an atomic operation.

\section{Algorithm 12 ITDsqI $(s q, o): s q^{\prime}$}

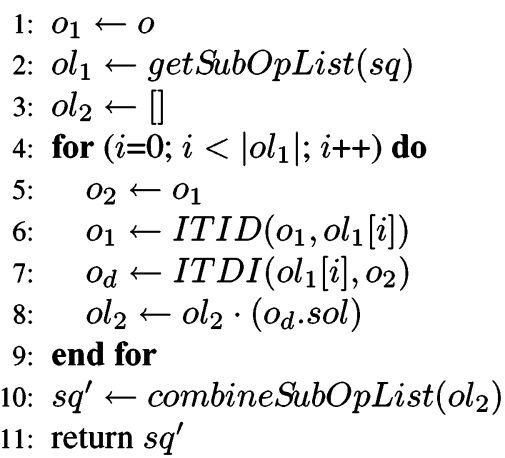

Next, we specify function ITDsqI(sq,o) for transforming a sequence $s q$ with an operation $o$ to incorporate the effects of $o$ into every operation in $s q$, as shown in Algorithm 12. Since ITDsqI is only called in Algorithm 2, we know that its input $s q$ is deletion-only and $o$ is but an insertion. A pitfall in implementing ITDsqI $(s q, o)$ is to naively transform every operation in $s q$ with $o$ one by one. The input precondition is $s q \sqcup o$, or $s q[0] \sqcup o$. Hence it makes sense to do IT $(s q[0], o)$. However, for the next operation, $s q[1]$, the relation is not $s q[1] \sqcup o$. Hence it makes no sense to do IT $(s q[1], o)$. The fix is to first transform $o$ with $s q[0]$, yielding $o^{\prime}$, and then transform $s q[1]$ with $o^{\prime}$. Following this idea, we first collect all sub-operations of $s q$ into list $o l_{1}$; for every operation in $o l_{1}$, we transform $o$ with $o l_{1}[i]$, and then transform $o l_{1}[i]$ with $o$, yielding $o_{d}$. All sub-operations in $o_{d}$ are collected in list $o l_{2}$. Finally, we combine the sub-operations and return a sequence of composite operations $s q^{\prime}$.

\subsection{SWAP Algorithms}

We first present the basic swap functions for swapping two primitive operations, and then discuss advanced swap functions that involve sequences of operations.

\subsubsection{Basic swap Functions}

Given two operations $o_{1}$ and $o_{2}$, where $o_{1} \mapsto o_{2}$, function $\operatorname{swap}\left(o_{1}, o_{2}\right)$ transposes them into $o_{1}^{\prime}$ and $o_{2}^{\prime}$ such that $o_{2}^{\prime} \mapsto o_{1}^{\prime}$. Depending on their types, insert (I) and delete 

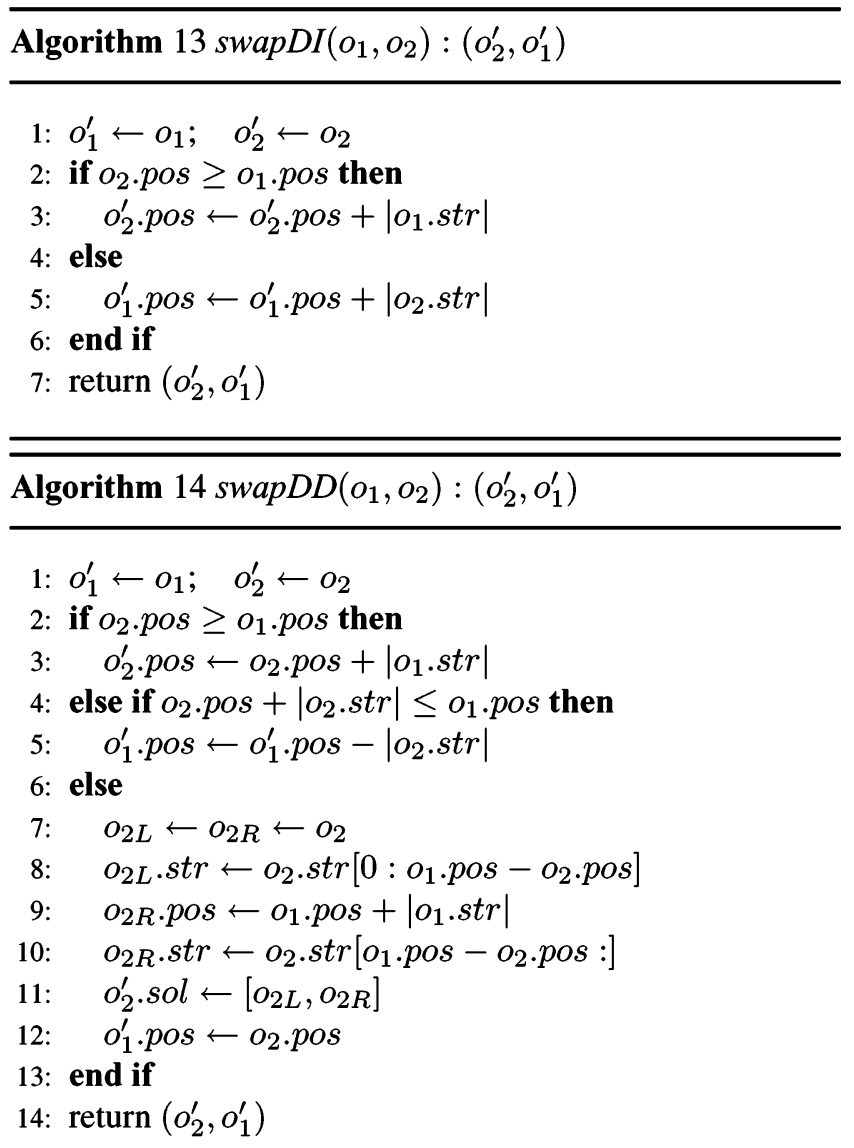

(D), we specify two basic swapping functions as in Algorithms 13 and 14, respectively. Function swapII is used in function transposeHC (called in Algorithm 2) and omitted altogether for space reasons. Function swapID is irrelevant because it is not used in our ABTS algorithm at all.

Algorithm 13 swaps a deletion $o_{1}$ and an insertion $o_{2}$. The case of $o_{2}$.pos $\geq o_{1}$.pos means that, after $o_{1}$ deletes $o_{1}$.str, $o_{2}$ inserts $o_{2} . s t r$ on the right side of the region $o_{1}$.str originally occupies. Hence, if they are swapped, meaning that $o_{2}$ inserts $o_{2} . s t r$ before $o_{1}$ deletes $o_{1} . s t r$, then $o_{2}$.pos should be shifted by $\mid o_{1}$.str $\mid$ characters to the right because $o_{1} . s t r$ is not deleted yet. The case of $o_{2}$.pos < $o_{1}$.pos means that, after $o_{1}$ deletes $o_{1} . s t r, o_{2}$ inserts $o_{2} . s t r$ on the strict left of $o_{1}$.pos and there are at least one character between $o_{2}$.pos and $o_{1}$.pos. Hence swapping the execution order of $o_{1}$ and $o_{2}$ entails that $o_{1}$.pos be shifted by $\left|o_{2} . s t r\right|$ characters to the right to account for $\mathrm{O}_{2} . \mathrm{str}$.

As specified in Algorithm 14, function $\operatorname{swapDD}\left(o_{1}, o_{2}\right)$ transposes two deletions $o_{1}$ and $o_{2}$. There are three cases to consider: First, if $o_{2} \cdot p o s \geq o_{1}$.pos, it means that $o_{2}$ is to delete a substring on the right side of the substring $o_{1}$.str deleted by $o_{1}$. Hence, if we execute $o_{2}$ before $o_{1}$ instead, then $o_{2}$.pos should account for $o_{1}$.str because it has not been deleted yet. Secondly, if $o_{2} . p o s+\left|o_{2} . s t r\right| \leq o_{1}$.pos, it means that $o_{2} . s t r$ is completely on the left side of $o_{1}$.pos.
Hence, if we execute $o_{2}$ before $o_{1}$ instead, $o_{1}$.pos should be shifted to the left because $o_{2} . s t r$ has been deleted. Thirdly, as in lines 6-12, o $o_{1} . s t r$ is completely covered by $o_{2} . s t r$. Then, if we execute $o_{2}$ before $o_{1}$ instead, $o_{2} . s t r$ is divided into three parts, among which the middle part is to be deleted by $o_{1}$. The remaining left and right parts, as divided by position $o_{1}$.pos, are deleted by two sub-operations $o_{2 L}$ and $o_{2 R}$, respectively. Finally, $o_{1}$.pos should be set to $\mathrm{o}_{2}$.pos due to the deletion of $o_{2 L} . s t r$.

\subsubsection{Sequence-Related swap Functions}



Algorithm 16 swapDsqD $(s q, o):\left(o^{\prime}, s q^{\prime}\right)$

1: $o^{\prime} \leftarrow o$

2: ol $\leftarrow$ getSubOpList $(s q)$

3: $\left(o^{\prime}\right.$. sol, ol $) \leftarrow \operatorname{swapLL}\left(\right.$ ol,$o^{\prime}$. sol,$\left.|o l|-1\right)$

4: $s q^{\prime} \leftarrow$ combineSubOpList $($ ol $)$

5: return $\left(o^{\prime}, s q^{\prime}\right)$

As in Algorithm 15, function swapDsqI $(s q, o)$ transposes a deletion sequence $s q$ with an insertion $o$, where $s q \mapsto o$, into $s q^{\prime}$ and $o^{\prime}$ such that $o^{\prime} \mapsto s q^{\prime}$. We first flatten $s q$ by collecting all sub-operations of $s q$ in list $o l$. Then we call the specified function $s w a p D I$ to transpose every operation in $o l$ with $o$ from right to left. Finally we merge all suboperations in $o l$ and return the resulting sequence as $s q^{\prime}$.

As in Algorithm 16, function $\operatorname{swapDsqD}(s q, o)$ transposes a deletion sequence $s q$ and a deletion $o$. The process is more complicated because swapping two deletions may result in composite operations. Similarly to Algorithm 14, we first flatten $s q$ into list $o l$, then call function $s w a p L L$ to transpose list $o l$ and list $o . s o l$, and finally combine suboperations in the resulting list. When calling swapLL, the third parameter is the index indicating from which operation in list $o l$ we start the actual swapping.

The algorithm for transposing two lists is implemented by a double recursion of two functions, swapLL and swapOL, as specified in Algorithm 17 and 18, respectively. Function $\operatorname{swapLL}\left(o l_{1}, o_{2}, p\right)$ transposes two given list $o l_{1}$ and $o l_{2}$, where $o l_{1} \mapsto o l_{2}$, into $o l_{1}^{\prime}$ and $o l_{2}^{\prime}$ such that 



$o l_{2}^{\prime} \mapsto o l_{1}^{\prime}$. Due to Algorithm 16, the input $o l_{2}$ is a suboperation list. That is, operations in $o l_{2}$ are defined relative to the same state. Hence the ordering of operations in $\mathrm{ol}_{2}$ is not important. Function swapLL works as follows: First, we call swapLO $\left(o l_{1}^{\prime}, o l_{2} . h e a d, p\right)$ to transpose $o l_{1}^{\prime}$ with the first operation in $o l_{2}$, where $o l_{1}^{\prime}$ is a copy of $o l_{1}$. While $o l_{1}^{\prime}$ is transformed in place, operation $o_{2}$.head is transformed into list $o l_{2 H}$. Then, we recursively call function swapLL $\left(o l_{1}^{\prime}, o l_{2} . t a i l, p\right)$ to transpose $o l_{1}^{\prime}$ with the remaining operations in $o l_{2}$. Again, while $o l_{1}^{\prime}$ is transformed in place, list $o l_{2}$.tail is transformed into list $o l_{2 T}$. Finally, we concatenate partial results $o l_{2 H}$ with $o l_{2 T}$ into $o l_{2}^{\prime}$ and return tuple $\left(o l_{2}^{\prime}, o l_{1}^{\prime}\right)$. Because operations in $o l_{2}$ are all contextually equivalent, operations in the resulting $o l_{2}^{\prime}$ are also contextually equivalent.

Function swapLO $\left(o l_{1}, o, p\right)$ transposes list $o l_{1}$ and operation $o$, where $o l_{1} \mapsto o$, into list $o l_{1}^{\prime}$ and list $o l_{2}^{\prime}$, respectively, such that $o l_{2}^{\prime} \mapsto o l_{1}^{\prime}$. Parameter $p$ points to the current operation in $o l_{1}$ to be transposed with $o$. If $p$ is zero, we call swapDD to transpose $o l_{1}[0]$ with $o$, yielding $o l_{1}^{\prime}[0]$ and $o^{\prime}$, and return the resulting $o^{\prime}$.sol and $o l_{1}^{\prime}$. Otherwise, we transpose every operation in $o l_{1}$ with $o$ from right to left, as in lines 5-8. First, we call $\operatorname{swapDD}\left(o l_{1}^{\prime}[p], o\right)$ to transpose the last operation in $o l_{1}^{\prime}$ and $o$, transforming $o$ into $o{ }^{\prime}$. Note that $o l_{1}^{\prime}$ is a copy of $o l_{1}$ and all its operations are transformed in place. As a result of swapDD, $o^{\prime}$ could be a composite operation. Hence we call swapLL $\left(o l_{1}^{\prime}, o^{\prime} . s o l, p-1\right)$ to transpose $o l_{1}^{\prime}$ with $o^{\prime} . s o l$, which transforms $o^{\prime}$.sol into $o l_{2}^{\prime}$.

\section{Analysis of Correctness}

ABTS is based on a well-proved theoretical framework called admissibility-based transformation (ABT) [6, 5], which establishes that an OT algorithm is correct if the following two formal conditions always hold:

(1) Causality preservation: whenever an operation $o$ is executed at a site, all operations that happen before $o$ must have been executed at that site.

(2) Admissibility preservation: the execution of every operation is admissible, i.e., it does not introduce inconsistent ordering of objects at different sites.

Condition (1) is satisfied by using vector timestamps. To satisfy condition (2), our approach is to first establish sufficient conditions of the basic IT and swap functions and then design a control procedure that satisfies those sufficient conditions while integrating local and remote operations. These two conditions together imply convergence $[6,5]$.

According to [11], the precondition of $\operatorname{IT}\left(o_{1}, o_{2}\right)$ is $o_{1} \sqcup$ $o_{2}$, and the precondition of $\operatorname{swap}\left(o_{1}, o_{2}\right)$ is $o_{1} \mapsto o_{2}$. In [6, $5]$, they are extended with the following proved sufficient conditions for the basic IT and swap functions to produce the correct results, i.e., admissible operations, assuming that $o_{1}$ and $o_{2}$ are admissible:

(a) IT $\left(o_{1}, o_{2}\right)$ is admissible if $o_{1} \sqcup o_{2}$ and, in the case they are both insertions and their positions tie, $o_{1} \| o_{2}$ and neither $o_{1}$ nor $o_{2}$ includes effects of any deletions.

(b) $\operatorname{swap}\left(o_{1}, o_{2}\right)$ is admissible if $o_{1} \mapsto o_{2}$ and, in the case $o_{1}$ is a deletion and $o_{2}$ is an insertion and their positions tie, $o_{1} \rightarrow o_{2}$ and $o_{2}$ is generated in state $\operatorname{dst}\left(o_{2}\right)$.

The presented ABTS algorithm has mainly two parts, updateHL and updateHR. The correctness of updateHL $(o)$ for integrating a local operation $o$ (Algorithm 1) is ensured as follows: when $o$ is a deletion, it is only swapped with deletions in $H_{d}$. At every step (Algorithms 16-18), as long as $o_{1} \mapsto o_{2}$ is guaranteed for every swapDD $\left(o_{1}, o_{2}\right)$, the result is correct. By discussions in Section 4.6, this is ensured. On the other hand, when $o$ is an insertion, it is swapped with deletions in $H_{d}$. However, since $o$ is a local operation that happens after $H_{d}$ and generated in its definition state, the above condition (b) holds for every swapDI function called. Hence, updateHL $(o)$ is correct.

The correctness of updateHR $(o)$ for integrating a remote operation (Algorithm 2) is ensured as follows: In line 1, function transposeHC $[10,6]$ transposes an insertion-only sequence $H_{i}$, which ultimately calls basic function swapII. By the above condition (b), the result is correct as long as $o_{1} \mapsto o_{2}$ is ensured every time swapII $\left(o_{1}, o_{2}\right)$ is called. In line 2 , the result of ITOSq is correct by the above condition (a) because, although IT happens between two concurrent insertions, neither of them includes effects of any deletions as a consequence of updateHL. In line 3 , the result is correct as long as two operations involved in every IT are contextu- 
ally equivalent. Similarly the result of line 5 is also correct. Hence, updateHR $(o)$ is also correct.

\section{Analysis of Complexities}

Note that the double-recursion presentation of Algorithms 9 and 16 is only for the sake of conceptual clarity. In the actual system, we rewrite them in a more efficient way to avoid the runtime overheads of recursions.

The space complexity of the presented ABTS algorithm is trivially $O(|H|)$. The time complexity of ABTS is in the same order of magnitude as that of its characterwise version $\mathrm{ABT}[6,3,5]$, which is not counterintuitive.

For space reasons, here we only give the results: The time complexity to integrate a local operation is $o\left(\left|o l_{d}\right|\right.$. $|o . s t r|)$, where $o l_{d}$ is the corresponding sub-operation list of $H_{d}$. When $m=|o . s t r|$ and the average length of deleted strings in the history, $c=\left|o l_{d}\right| /\left|H_{d}\right|$ can be considered as small constants, the complexity is linear in the number of deletions in the history, i.e., $O\left(\left|H_{d}\right|\right)$. The execution of a remote operation $o$ takes time $O\left(\left|H_{i}\right|^{2}+\left(\left|s q_{c}\right|+\left|o l_{d}\right|\right)\right.$. $|o . s t r|)$, where $s q_{c}$ is the operations in $H_{i}$ that are concurrent with $o$ and $o l_{d}$ is the corresponding sub-operation list of $H_{d}$. When $m=|o . s t r|$ and $c=\left|o l_{d}\right| /\left|H_{d}\right|$ can be considered as small constants, the complexity is $O\left(\left|H_{i}\right|^{2}+\left|H_{d}\right|\right)$, roughly quadratic in the number of insertions in the history.

\section{Conclusions}

This paper presents a novel transformation based consistency control algorithm called ABTS that supports stringbased primitive operations. The presented algorithm is the first of its kind with stringwise operations and correctness formally proved. For space reasons, we only sketched the correctness proofs and the complexity analyses in this paper. Since operations are stored in their execution order in the history $H$, the time complexity to integrate a remote operation is roughly $O\left(|H|^{2}\right.$ ), which is in the same order of magnitude as its character-based precursor, ABT [6,5]. Although ABTS is extended from ABT, the extension is theoretically significant due to the complications in handling operation region overlapping and splitting. Moreover, the extension makes it possible to apply OT techniques to a wider range of practical collaborative applications.

In future research, we plan to extend this work specifically for application domains such as collaborative software development and study its usability. With support of string operations as the new starting point, it will be interesting to study techniques for conflicts detection and resolution in the context of specific application domains [11]. Another interesting direction is to optimize the algorithm to reduce the time complexity for it to work more efficiently for both real-time and asynchronous collaborative applications [3].

\section{Acknowledgments}

The work is supported in part by the National Natural Science Foundation of China (NSFC) under Grant No. 60736020 and No. 60803118, the Shanghai Science \& Technology Committee Key Fundamental Research Project under Grant No. 08JC1402700 and the Shanghai Leading Academic Discipline Project under Grant No. B114.

\section{References}

[1] C. A. Ellis and S. J. Gibbs. Concurrency control in groupware systems. In Proceedings of the ACM SIGMOD'89 Conference on Management of Data, pages 399-407, Portland Oregon, 1989.

[2] L. Lamport. Time, clocks, and the ordering of events in a distributed system. Communications of the ACM, 21(7):558-565, July 1978.

[3] D. Li and R. Li. A performance study of group editing algorithms. In The 12th International Conference on Parallel and Distributed Systems (ICPADS'06), pages 300-307, Minneapolis, MN, July 2006.

[4] D. Li and R. Li. An approach to ensuring consistency in peer-to-peer real-time group editors. Computer Supported Cooperative Work: The Journal of Collaborative Computing, 17(5-6):553-611, Dec. 2008.

[5] D. Li and R. Li. An admissibility-based operational transformation framework for collaborative editing systems. Computer Supported Cooperative Work: The Journal of Collaborative Computing, Aug. 2009. Accepted.

[6] R. Li and D. Li. Commutativity-based concurrency control in groupware. In Proceedings of the First IEEE Conference on Collaborative Computing: Networking, Applications and Worksharing (CollaborateCom'05), San Jose, CA, Dec. 2005.

[7] R. Li and D. Li. A new operational transformation framework for real-time group editors. IEEE Transactions on Parallel and Distributed Systems, 18(3):307-319, Mar. 2007.

[8] G. Oster, P. Urso, P. Molli, and A. Imine. Proving correctness of transformation functions in collaborative editing systems. Technical Report 5795, INRIA, Dec. 2005.

[9] M. Suleiman, M. Cart, and J. Ferrié. Concurrent operations in a distributed and mobile collaborative environment. In IEEE ICDE'98 International Conference on Data Engineering, pages 36-45, Feb. 1998.

[10] C. Sun and C. Ellis. Operational transformation in real-time group editors: issues, algorithms, and achievements. In Proceedings of the ACM Conference on Computer-Supported Cooperative Work, pages 59-68, Dec. 1998.

[11] C. Sun, X. Jia, Y. Zhang, Y. Yang, and D. Chen. Achieving convergence, causality-preservation, and intentionpreservation in real-time cooperative editing systems. $A C M$ Transactions on Computer-Human Interaction, 5(1):63108, Mar. 1998.

[12] D. Sun and C. Sun. Context-based operational transformation in distributed collaborative editing systems. IEEE Transactions on Parallel and Distributed Systems, 20(10):1454-1470, 2009. 